\title{
Malignant Perivascular Epithelioid Cell Tumor of the Uterus with Lung Metastasis
}

\author{
Jeong Bae Kang · Jin Won Seo ${ }^{1}$ Y Young-Han Park ${ }^{2}$ Pong Rheem Jang \\ Department of Obstetrics and Gynecology, Dongtan Sacred Heart Hospital, Hallym University College of Medicine, Hwaseong; Departments of ${ }^{1}$ Pathology and \\ ${ }^{2}$ Obstetrics and Gynecology, Hallym University Sacred Heart Hospital, Hallym University College of Medicine, Anyang, Korea
}

Perivascular epithelioid cell tumor (PEComa) is an extremely rare mesenchymal tumor, which can arise in a wide array of anatomic locations, and is characterized by its myelomelanocytic phenotype and unpredictable natural history. Specifically, PEComas, which occur in non-classic anatomic distributions, are known as PEComa-not otherwise specified (PEComa-NOS). ${ }^{1}$ To the best of our knowledge, a total of 234 cases of PEComasNOS have been reported in English published reports. Of those, 47 were uterine PEComas cases, which included 31 tumors of benign behavior and 16 tumors of locally aggressive behavior with distant metastasis to liver, lungs, intestines, bone, and lymph nodes. Here, we report a very rare malignant uterine PEComa with lung metastasis in a 49-year-old unmarried woman, despite adjuvant chemotherapy after initial surgery.

\section{CASE REPORT}

A 49-year-old unmarried woman visited a gynecologist complaining of her abnormal uterine bleeding, which had lasted for 1 week. Her past medical history was unremarkable. Upon vaginal examination, the uterus was slightly enlarged and there was a moderate tenderness in the lower abdomen. Ultrasonography showed a $9.5 \mathrm{~cm} \times 8.0 \mathrm{~cm} \times 7.5 \mathrm{~cm}$-sized tumor with high vascularity, arising from the anterior wall of the uterine corpus. Vital signs were within normal range. The initial laboratory find-

\section{Corresponding Author}

Young-Han Park, M.D.

Department of Obstetrics and Gynecology, Hallym University Sacred Heart Hospital, Hallym University College of Medicine, 22 Gwanpyeong-ro 170beon-gil, Dongan-gu, Anyang 431-796, Korea

Tel: +82-31-380-3820, Fax: +82-31-380-1900, E-mail: drparkyh@yahoo.co.kr

Received: March 27, 2014 Revised: June 17, 2014

Accepted: June 19, 2014 ings were normal, except for a low hematocrit level of $28 \%$. The chest X-ray showed no active lung lesions (Fig. 1A). Under the diagnosis of uterine leiomyoma, she was taken to the operative room the following day. During the explorative laparotomy, a large, fungating, vascular mass arising from the anterior wall of the uterine corpus was noted. Myomectomy was performed because of her desire to preserve the uterus. The specimen was sent to a pathologist for frozen section. The pathologist conformed that the mass was highly suspicious for malignancy with high mitotic figures and marked nuclear anaplasia. Consequently, a total abdominal hysterectomy and bilateral salpingo-oophorectomy was performed. On gross examination, the tumor was an ovoid firm myomatous nodule $(8.5 \times 8.0 \times 6.5 \mathrm{~cm})$. On section, the cut surface of the mass showed yellow white fish-fleshy appearance with multifocal necrosis (Fig. 2A). Microscopic review revealed that the tumor cells were predominantly composed of epithelioid cells with eosinophilic cytoplasm. Also, an extensive area of trabecular arrangement, marked nuclear anaplasia, necrosis and vascular invasion was noted. Twenty mitotic figures per 10 high-power fields were observed (Fig. 2B). In immunohistochemical staining, the neoplastic cells were strongly positive for human melanoma black 45 (HMB-45) (Fig. 2C) and smooth muscle actin (SMA) (Fig. 2D), whereas they were negative for $\mathrm{p} 53$, desmin, creatine kinase, c-kit, and CD10. In combination of these pathological features and the myelomelanocytic phenotype of the tumor, the final diagnosis was uterine PEComa. Considering the large size of the tumor and the presence of multiple high risk features as proposed by Folpe et al. ${ }^{2}$ a malignant or high-risk PEComa was suspected (Table 1). After surgery, the patient underwent three sessions of adjuvant chemotherapy (doxorubicin hydrochloride+haloxan) and was followed up regularly. Seven months later, the patient was taken 

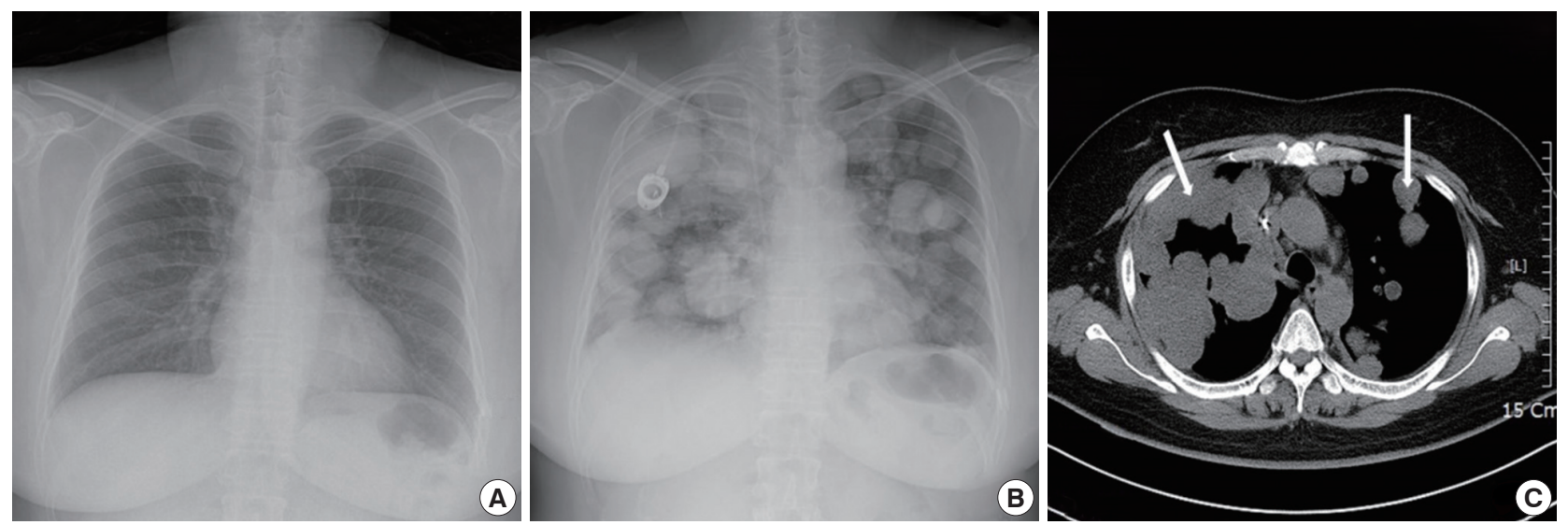

Fig. 1. Radiologic finding of pulmonary metastasis. (A) Initial finding of chest $\mathrm{X}$-ray shows no active lesion. Chest X-ray (B) and computed tomography scan (C) after seven months show multiple round nodules with variable sizes in both lungs (arrows).
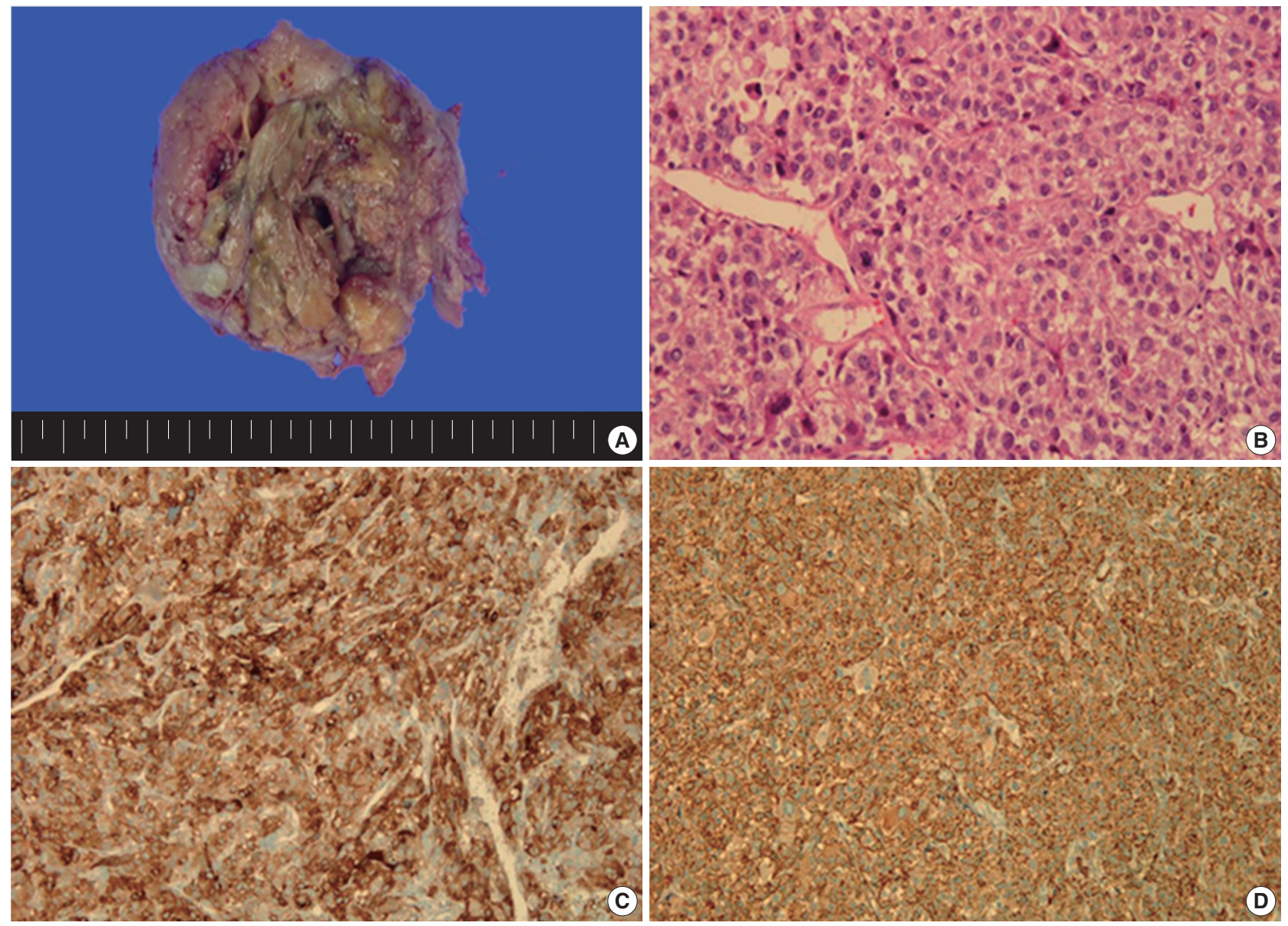

Fig. 2. Gross and microscopic findings of the specimen. (A) The tumor is a round mass $(8.5 \times 8.0 \times 6.5 \mathrm{~cm})$. On section, the cut surface shows yellow white fish-fleshy appearance with multifocal necrosis. (B) Microscopic findings reveal that the tumor cells are composed of epithelioid cells with eosinophilic cytoplasm, trabecular arrangement, marked nuclear atypia, necrosis, vascular invasion, and frequent mitosis. The neoplastic cells are strongly positive for HMB-45 (C) and SMA (D) in immunohistochemical staining.

to an emergency room with general weakness, high fever $\left(38.6^{\circ} \mathrm{C}\right)$ and dyspnea. The chest X-ray and computed tomography (CT) findings showed multiple round nodules with variable sizes in both lungs which were suspected as metastasis (Fig. 1B, C). Af- 
Table 1. Proposed classification of PEComas

\begin{tabular}{l} 
Characteristic \\
\hline High risk features \\
Size $>5 \mathrm{~cm}$ \\
Infiltrative growth pattern \\
High nuclear grade and cellularity \\
Mitotic rate $>1 / 50$ high-power field \\
Necrosis \\
Vascular invasion \\
Risk category \\
Benign: $<2$ high risk features and size $<5 \mathrm{~cm}$ \\
Uncertain malignant potential: size $\geq 5 \mathrm{~cm}$ with no other high risk \\
features or nuclear pleomorphism $/$ multinucleated giant cells only \\
Malignant: 2 or more high risk features
\end{tabular}

Adapted from Folpe et al. Am J Surg Pathol 2005; 29: 1558-75, ${ }^{2}$ with permission from Wolters Kluwer Health.

PEComa, perivascular epithelioid cell tumor.

ter admission, the patient started chemotherapy again with the same drugs as previously used, but expired during the treatment due to pneumonia. The patient's family gave an informed consent to the publication.

\section{DISCUSSION}

The perivascular epithelioid cell was first addressed by Bonetti et al. in 1992, ${ }^{3}$ according to its morphological and immunohistochemical features. Subsequently, they further proposed that the diseases with the same phenotype, including angiomyolipoma (AML), clear cell "sugar" tumor (CCST) of the lung, and lymphangioleiomyomatosis (LAM), be a panel of entity. Then, in 1996, PEComa was proposed by Zamboni et al. ${ }^{4}$ The PEComa family of tumors comprised of AML, CCST of the lung, LAM, and less well-characterized PEComas of other anatomic origins, for which the term PEComa-NOS has been proposed. PEComa-NOS is a collection of rare tumors defined as a mesenchymal tumor composed of perivascular epithelioid cells with distinctive morphological and immunohistochemical features. ${ }^{5}$ PEComa-NOS has now been reported in almost every body site, including gynecological, genitourinary, gastrointestinal, extremities, skin, heart, breast, oral cavity, orbit, and skull base. Specifically, uterus is the most prevalent site of PEComa-NOS. ${ }^{5}$ Most of the patients with uterine PEComas show abnormal uterine bleeding or lower abdominal pain with a palpable mass, which is similar to the symptoms and signs of other uterine tumors. Thus, uterine PEComas can often be misdiagnosed as uterine leiomyomas before surgery. Most clinical courses of uterine PEComa are benign, and malignant tumors are rare. Furthermore, distant metastasis to the lung in malignant PEComa of the uterus is extremely rare. Distant metastatic diseases of uter- ine PEComas are only 6.8\% (16/234) of all cases. The most common location of distant metastasis was lung ( 7 cases), which was followed by bone ( 5 cases), ovary, liver, adrenal gland and peritoneal wall. The risk of metastasis depends on the size of the primary mass and the presence of multiple high risk features. ${ }^{2}$ CT findings are pathognomonic, showing multiple round nodules of variable sizes distributed throughout the lungs, including the costophrenic angles, with normal intervening lung parenchyma. ${ }^{6}$ Since PEComa has no distinctive symptoms and signs, the diagnosis should depend on pathological examinations. PEComas are usually composed of epithelioid, but occasionally spindled cells, with clear to granular eosinophilic cytoplasm and focal perivascular accentuation. ${ }^{7}$ In our case, the tumor cells were predominantly composed of epithelioid cells with eosinophilic cytoplasm. In addition, mitotic figures and necrosis can be observed. Thin-walled blood vessels are readily visible around the smooth muscle with unclear boundaries. Also in immunohistochemical staining, these tumors are positive for markers of both melanocytic (HMB-45, Melan-A, and tyrosinase) and myoid (desmin, SMA, muscle-specific actin, caldesmon, and calponin) differentiation. ${ }^{8}$ Schoolmeester $e t$ al. ${ }^{9}$ reported that HMB-45 in PEComas is the most sensitive (16/16 positive, 100\%) marker, followed by MiTF (11/12 positive, $92 \%$ ) and Melan-A (14/16 positive, 88\%); all three muscle markers were expressed by most tumors in that study. Desmin was positive in 15 of 15 cases (100\%), SMA was positive in 14 of 15 cases (93\%), and h-caldesmon was positive in 11 of 12 cases (92\%). ${ }^{9}$ Fadare reported that PEComas are immunoreactive for melanocytic markers and most frequently, HMB-45. Furthermore, 49\% (19/35 cases) and 73\% (24/33 cases) of PEComas are immunohistochemically positive for desmin and SMA, respectively. ${ }^{10}$ In our case, the tumor showed strong and diffuse positivity for both HMB-45 and SMA in immunohistochemical staining, whereas it was negative for $\mathrm{p} 53$, desmin, and CD10.

The uterine PEComas should be differentiated from other uterine tumors, such as epithelioid leiomyoma, leiomyosarcoma and endometrial stromal sarcoma (ESS). ${ }^{6}$ These tumors and PEComa can share, at least in part, some histological appearances and immunoprofiles, including expression of melanocytic and smooth muscle markers. ${ }^{7}$ Epithelioid leiomyoma shows focal or no immunoreactivity for HMB-45, with positivity for SMA and desmin. HMB-45 positivity, often focal, has been found in $31 \%$ to $36 \%$ of conventional leiomyosarcomas, but leiomyosarcoma without clear cell areas has been found to be negative for HMB-45. ' Immunoreactivity for HMB-45 has been found only in clear cell areas. ESS shows negative staining for HMB-45, 
SMA, and desmin. ${ }^{10}$ Therefore, the distinction between PEComas and epithelioid smooth muscle tumors should be largely a morphologic exercise: the latter lacks the vascular network of the former and will typically show at least focal conventional areas. ${ }^{910}$ Although PEComas have a wide spectrum of biological behavior, convincing criteria for malignancy have not yet been established. As mentioned earlier, a proposed classification of PEComas adapted from Folpe et al. ${ }^{2}$ has been widely used up to now. Our case, a large tumor with marked nuclear anaplasia, necrosis, high mitotic figures, and vascular invasion of the tumor cells, conforms to the malignant or high-risk PEComa according to the proposed classification. Optimal treatment for PEComas has not been well established at this time. Currently, surgery is the mainstay of treatment. Although radiotherapy, chemotherapy or immunotherapy is being implemented as an adjuvant treatment for malignant PEComa after surgery, the actual effect has not been well demonstrated. Because malignant PEComa portends a poor prognosis, treatment strategies incorporating surgery, radiotherapy and chemotherapy should be considered.

\section{Conflicts of Interest}

No potential conflict of interest relevant to this article was reported.

\section{REFERENCES}

1. Fadare O, Parkash V, Yilmaz Y, et al. Perivascular epithelioid cell tumor (PEComa) of the uterine cervix associated with intraabdominal "PEComatosis": a clinicopathological study with comparative genomic hybridization analysis. World J Surg Oncol 2004; 2: 35.
2. Folpe AL, Mentzel T, Lehr HA, Fisher C, Balzer BL, Weiss SW. Perivascular epithelioid cell neoplasms of soft tissue and gynecologic origin: a clinicopathologic study of 26 cases and review of the literature. Am J Surg Pathol 2005; 29: 1558-75.

3. Bonetti F, Pea M, Martignoni G, Zamboni G. PEC and sugar. Am J Surg Pathol 1992; 16: 307-8.

4. Zamboni G, Pea M, Martignoni G, et al. Clear cell "sugar" tumor of the pancreas: a novel member of the family of lesions characterized by the presence of perivascular epithelioid cells. Am J Surg Pathol 1996; 20: 722-30.

5. Folpe AL. Neoplasms with perivascular epithelioid cell differentiation (PEComas). In: Fletcher CD, Unni KK, Mertens F, eds. World Health Organization classification of tumors: pathology and genetics of tumors of soft tissue and bone. Lyon: IARC Press, 2002; 221-2.

6. Bleeker JS, Quevedo JF, Folpe AL. "Malignant” perivascular epithelioid cell neoplasm: risk stratification and treatment strategies. Sarcoma 2012; 2012: 541626.

7. Ye HY, Chen JG, Luo DL, Jiang ZM, Chen ZH. Perivascular epithelioid cell tumor (PEComa) of gynecologic origin: a clinicopathological study of three cases. Eur J Gynaecol Oncol 2012; 33: 105-8.

8. Yamada Y, Yamamoto H, Ohishi Y, et al. Sclerosing variant of perivascular epithelioid cell tumor in the female genital organs. Pathol Int 2011; 61: 768-72.

9. Schoolmeester JK, Howitt BE, Hirsch MS, Dal Cin P, Quade BJ, Nucci MR. Perivascular epithelioid cell neoplasm (PEComa) of the gynecologic tract: clinicopathologic and immunohistochemical characterization of 16 cases. Am J Surg Pathol 2014; 38: 176-88.

10. Fadare O. Perivascular epithelioid cell tumor (PEComa) of the uterus: an outcome-based clinicopathologic analysis of 41 reported cases. Adv Anat Pathol 2008; 15: 63-75. 